\title{
Compact Nonlinear Springs with User Defined Torque-Deflection Profiles for Series Elastic Actuators
}

\author{
Alexander Schepelmann ${ }^{1}$, Kathryn A. Geberth ${ }^{1}$, and Hartmut Geyer ${ }^{1}$
}

\begin{abstract}
Series elastic actuators often use linear metal springs in their drivetrains, which requires design compromises between torque resolution and actuation bandwidth. Nonlinear springs (NLSs), with variable stiffness, overcome this limitation, enabling both high torque resolution and high bandwidth. Current NLS designs combine variable cam structures with off-the-shelf linear springs, which increases the overall size of these torque transmitting elements. NLS size could be reduced by using other materials as an elastic element. We present an optimization-based synthesis method for NLSs that are compact and encode a user-defined torque-deflection profile using elastic elements with an arbitrary stiffness profile. We experimentally validate the proposed method by creating a NLS prototype and testing it on an actuator testbed. The prototype uses rubber as the elastic element, resulting in a compact design that generates the desired torque profile, although hysteresis of the rubber material partially compromises performance. The results suggest that the proposed method successfully generates compact NLS designs, but that rubber elements need to be carefully chosen to mitigate hysteresis.
\end{abstract}

\section{INTRODUCTION}

Series elastic actuators (SEAs) often use linear metal springs as the torque transmitting elements in their drivetrains [1][2][3][4]. As commercial, off-the-shelf products, metal springs can be easily bought at low prices. However, using series linear springs in the drivetrain requires compromises between torque resolution and actuation bandwidth [5][6]. Torque resolution describes the smallest change in torque that can be measured. It is a function of spring stiffness, with softer springs providing higher torque resolution. Bandwidth describes the speed at which an actuator can apply torque to a load [7]. It can be characterized by rise time, the time an actuator takes to apply its maximum torque to a load when starting from rest, which, for SEAs, also depends on the series spring stiffness. Softer springs cause longer rise times, resulting in lower actuation bandwidth.

In contrast to linear springs, nonlinear torque transmitting elements with variable stiffness enable both high torque resolution and high bandwidth. For instance, variable stiffness actuators enable a range of torque resolutions and actuation bandwidths with a single actuator by actively tuning a passive mechanical element with a secondary motor [8][9][10][11]. However, such actuators are mechanically complicated, large, and heavy, hampering their application in small, lightweight robots, as well as for retrofit in existing robots.

\footnotetext{
*This work is supported by the Eunice Kennedy Shriver National Institute of Child Health \& Human Development under award no. 1R01HD075492. A. S. is supported by the NSF GRFP Program under grant 0946825.

${ }^{1}$ A. Schepelmann, K. A. Geberth, and H. Geyer are with the Robotics Institute, Carnegie Mellon University, Pittsburgh, PA 15213, USA. \{aschepelmann, kgeberth, hgeyer\} @cmu.edu
}

SEAs with purely passive nonlinear springs (NLSs) are a subset of variable stiffness actuators that omit active tuning of the mechanical element at the cost of embedding a single nonlinear torque-deflection profile. This profile can be designed to meet desired torque resolution and rise time goals. Different realizations of this idea have been pursued, many of which deflect linear metal springs with cams of changing radius. Early designs favored mechanically complex devices for mathematical simplicity, where the cam radius and spring force vectors are kept perpendicular throughout the NLS deflection [12][13]. More recently, NLS designs have been simplified mechanically at the cost of mathematical complexity. In [14], the authors identified a closed-form solution to cam profiles for which a linear spring wraps around the cam. While the assumption of a linear spring was necessary to obtain the solution, it does not fully explore the potential for miniaturizing passive nonlinear springs, as the cam profile was again designed around commercially available spring form factors and stiffnesses [15].

Rubber is an alternative to commercial linear metal springs as the elastic element in NLS designs. Although rubber has some disadvantages when compared to metal springs, such as increased hysteresis and van der Waal force-dependent stiffness characteristics [16][17], it tolerates large stretches before plastic deformation and can be molded with custom form factors. Due to these advantages, rubber is already being used in SEAs when actuator size matters [18]. In contrast to metal springs, rubber springs can be nonlinear, especially at large stretches [19]. Therefore, the closed form solution for NLS designs developed in [14] cannot be applied.

To overcome this limitation, we propose and demonstrate an optimization-based synthesis method for compact NLSs that generate desired nonlinear torque profiles. In section II, we describe the optimization procedure to generate NLS designs. Using a particular NLS design as an example, we outline the manufacturing process of an NLS prototype in section III. In section IV, we characterize the resulting prototype via experiments. The experiments show that custom torque profiles can be realized with a small form factor. They also point to drawbacks that stem from using rubber material as the elastic element. We discuss future steps to overcome these drawbacks in section V.

\section{NONLINEAR SPRING OPTIMIZATION}

The proposed NLS design is a two part assembly, consisting of an elastic element and a rotary cam whose profile is optimized to stretch the elastic element over a variable radius (Fig. 1). The cam is defined by a set of $(x, y)$ pairs. 


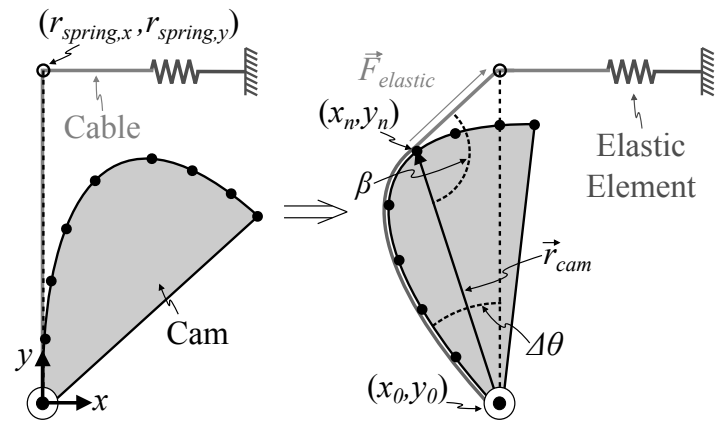

Fig. 1. NLS concept. L: Undeflected spring. R: Spring deflected $\Delta \theta$. A cam engages a cable attached to an elastic element as it rotates about $\left(x_{0}, y_{0}\right)$, stretching the elastic element. The cross product of the radius from $\left(x_{0}, y_{0}\right)$ and the last contact point between the cable and cam $\left(x_{n}, y_{n}\right)$ with the force generated by the elastic element's stretch creates a desired torque $\tau_{d e s}$.

The optimization uses evenly spaced torque-deflection pairs of desired torques $\tau_{\text {des }}$ for spring deflections $\Delta \theta$

$$
\left[\begin{array}{c}
\tau_{\text {des }} \\
\Delta \theta
\end{array}\right]=\left[\begin{array}{c}
0 \cdots \tau_{\text {des }, \max } \\
0 \cdots \Delta \theta_{\max }
\end{array}\right]
$$

where $\tau_{\text {des, } \max }$ and $\Delta \theta_{\max }$ are the maximum desired torque and spring deflection, respectively, and the elastic element's force-displacement relationship

$$
\boldsymbol{F}_{\text {elastic }}=f(\Delta s)
$$

where $\Delta s$ is the change in the elastic element's length due to cam engagement. $\Delta s$ is the sum of the Euclidian distance between points on the cam with the distance between the cam's last contact point and attachment point to the NLS housing, minus the elastic element's rest length. $\Delta s$ occurs as convex points on the cam $\left(x_{i}, y_{i}\right)$ engage the elastic element throughout the cam rotation, and is given by

$$
\begin{aligned}
\Delta s= & \sum_{i=1}^{n} \sqrt{\left(x_{i}-x_{i-1}\right)^{2}+\left(y_{i}-y_{i-1}\right)^{2}}+ \\
& \sqrt{\left(r_{\text {spring }, x}-x_{n}\right)^{2}+\left(r_{\text {spring }, y}-y_{n}\right)^{2}}- \\
& \sqrt{\left(r_{\text {spring }, x}-x_{0}\right)^{2}+\left(r_{\text {spring }, y}-y_{0}\right)^{2}}
\end{aligned}
$$

where $\left(x_{n}, y_{n}\right)$ is the last, tangent contact point between the cam and elastic element and $\left(r_{\text {spring, }}, r_{\text {spring,y }}\right)$ is the elastic element's contact point with the NLS housing. This latter parameter is based on the desired NLS diameter.

To optimize the cam profile, the covariance matrix adaptation evolution strategy (CMA-ES) algorithm [20] is used. The optimization modifies $(x, y)$ pairs of the cam to minimize the difference between $\tau_{\text {des }}$ and the cam profile's current torque $\tau_{N L S}$ using the cost function

$$
J\left(\boldsymbol{\tau}_{N L \boldsymbol{S}}(\Delta \theta)\right)=\sum_{j=1}^{m}\left(\boldsymbol{\tau}_{\text {des }}(\Delta \theta)_{j}-\boldsymbol{\tau}_{\boldsymbol{N} \boldsymbol{L} \boldsymbol{S}}(\Delta \theta)_{j}\right)^{2}
$$

where

$$
\tau_{N L S}(\Delta \theta)_{j}=\boldsymbol{r}_{\text {cam }}(\Delta \theta)_{j} \times \boldsymbol{F}_{\text {elastic }}(\Delta \theta)_{j}
$$

Input: Cam profile

Calculate convex hull of cam profile;

for $\Delta \theta_{j}=0$ to $\Delta \theta_{\max }$ do

Rotate profile to $\Delta \theta_{j}$

Find $\left(x_{n}, y_{n}\right)$ between convex hull and elastic

element;

Calculate $\Delta s$;

Calculate $F_{\text {elastic }}(\Delta s)$;

Calculate $\tau_{N L \boldsymbol{S}}(\Delta \theta)_{j}$

end

$J\left(\boldsymbol{\tau}_{\boldsymbol{N} L \boldsymbol{S}}(\Delta \theta)\right)=\sum_{j=1}^{m}\left(\boldsymbol{\tau}_{\text {des }}(\Delta \theta)_{j}-\boldsymbol{\tau}_{N \boldsymbol{L} \boldsymbol{S}}(\Delta \theta)_{j}\right)^{2} ;$

Algorithm 1: Cam optimization criterion.

and $\boldsymbol{r}_{c a m}(\Delta \theta)_{j}$ is the vector from the cam's center of rotation $\left(x_{0}, y_{0}\right)$ to $\left(x_{n}, y_{n}\right)$ at the rotation $\Delta \theta_{j}$. The direction of $\boldsymbol{F}_{\text {elastic }}$ is defined by $\left(x_{n}, y_{n}\right)$ and $\left(r_{\text {spring, }}, r_{\text {spring,y }}\right)$. During each iteration, the optimization criterion (Alg. 1) first calculates the convex hull of the current cam profile. The cam profile then incrementally rotates through the deflection range. At each rotation, $\left(x_{n}, y_{n}\right)$ is found using line intersection; starting at $\left(x_{0}, y_{0}\right)$, the criterion checks for line intersections between $\boldsymbol{F}_{\text {elastic }}$ and subsequent points on the cam profile. $\left(x_{n}, y_{n}\right)$ is found when only one intersection exists. Next, the elastic element's resulting length due to cam rotation is calculated as the difference between the elastic element's rest length and the sum of the Euclidian distance between sequential points from $\left(x_{0}, y_{0}\right)$ to $\left(x_{n}, y_{n}\right)$ and $\left(x_{n}, y_{n}\right)$ to $\left(r_{\text {spring, } x}, r_{\text {spring }, y}\right)$. From this, $\boldsymbol{F}_{\text {elastic }}$ is evaluated, followed by $\tau_{N L S}(\Delta \theta)_{j}$. After rotating through the deflection range, $J\left(\boldsymbol{\tau}_{N L \boldsymbol{S}}(\Delta \theta)\right)$ is evaluated, and the optimization generates a new cam profile, finally resulting in a set of $(x, y)$ pairs that define the cam profile.

\section{NLS Manufacturing PRocess}

Here we describe the NLS manufacturing process. We first determine our desired torque-deflection profile via optimization based on design requirements. Next we select a rubber as our elastic element, characterize its force-deflection relationship, and manufacture a NLS prototype. Key components of the prototype are sized to meet geometric requirements, but are evaluated on a benchtop setup for ease of testing.

\section{A. Torque Profile Optimization}

We target NLSs for SEAs in a neuromuscular gait testbed [21]. The NLSs for these SEAs must generate an exponentially stiffening torque with a zero torque at zero deflection, and a maximum torque $\tau_{\max }=5 \mathrm{Nm}$ at $\Delta \theta=\Delta \theta_{\max }$. As a function of these constraints, the NLS torque profile is

$$
\tau_{\text {des }}\left(\Delta \theta, \tau_{\max }, \Delta \theta_{\max }\right)=\frac{\tau_{\max }}{e^{\Delta \theta_{\max }}-1}\left(e^{\Delta \theta}-1\right) .
$$

We desire a rise time of $t_{\text {rise }}=16 \mathrm{~ms}$, and a spring diameter less than $5 \mathrm{~cm} . \Delta \theta_{\max }$ is defined by the rise time. To meet $t_{\text {rise }}, \Delta \theta_{\max }$ is optimized by numerically solving the motor dynamics equation

$$
J_{m} \ddot{\theta}_{m}=\tau_{\text {motor }}-\tau_{\text {des }}\left(\Delta \theta, \tau_{\max }, \Delta \theta_{\max }\right)
$$




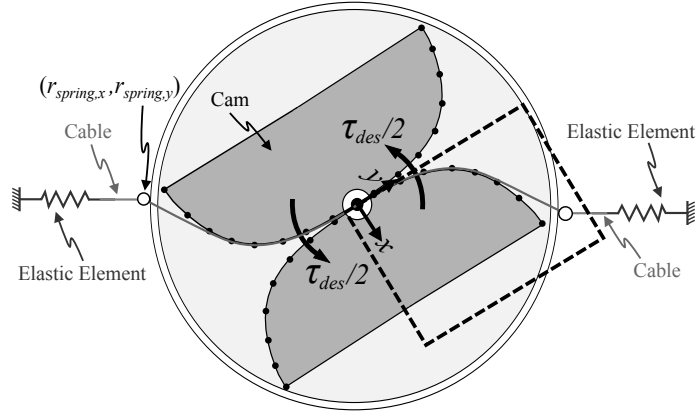

Fig. 2. NLS prototype schematic. The NLS is realized with two pieces: a cam and an outer housing, to which the cable and elastic element are attached. The elastic element stretches when the cable engages the cam as the two pieces rotate relative to each other. One elastic element is attached to each cable end; each cam surface generates $\tau_{d e s} / 2$. Dashed: Cam found via optimization to generate $\tau_{\text {des }} / 2$. The cam is mirrored about $y=-x$ to generate the full $\tau_{\text {des }}$ during spring deflection in one direction. These two cams are mirrored about $x=0$ to create a NLS with a symmetric torque-deflection profile about $\Delta \theta=0$

TABLE I

NLS OPTIMIZATION PARAMETERS \& RESUlTS

\begin{tabular}{|c|c|c|}
\hline $\boldsymbol{J}_{\boldsymbol{m}}\left(\mathrm{kgm}^{2}\right)$ & $\boldsymbol{t}_{\text {rise }}^{*}(\mathrm{~ms})$ & $\boldsymbol{\Delta} \boldsymbol{\theta}_{\boldsymbol{m a x}}\left(^{o}\right)$ \\
\hline $8.83 \times 10^{-5}$ & 16 & 53 \\
\hline
\end{tabular}

using constrained nonlinear optimization (MATLAB fmincon, active-set algorithm), where $J_{m}$ is the reflected SEA inertia at the spring, $\theta_{m}$ is the motor angle, $\tau_{\text {motor }}$ is the applied motor torque, and the actuator is assumed to start with no spring deflection from rest $\Delta \theta(0)=\dot{\theta}_{m}(0)=0$. In this case, $\Delta \theta$ is given by $\theta_{m}$, as the output shaft is assumed to be clamped. $J_{m}$ is estimated as the total reflected inertia of the motors, gears, and cam; inertia from encoders, shafts, and fasteners are ignored. During each iteration, the optimization numerically solves equation 7 via a fourth order Runge-Kutta method, modifying $\Delta \theta_{\max }$ to minimize the cost function

$$
J\left(t_{\text {rise }}^{*}\right)=\left(t_{\text {rise }}-t_{\text {rise }}^{*}\right)^{2}
$$

where $t_{\text {rise }}^{*}$ is the rise time for the current value of $\Delta \theta_{\max }$. With an optimized $\Delta \theta_{\text {max }}$, equation 6 now describes a spring profile that encodes the desired spring shape and rise time. Optimization parameters and results are listed in table I.

\section{B. Rubber Selection}

The NLS design is realized with parallel elastic elements, where each element and cam generate $\tau_{\text {des }} / 2$ (Fig. 2).

We use rubber as the elastic element in the NLS due to its loading properties and customizable form factor. Rubber dimensions must be selected to withstand the uniaxial tension applied by the cam throughout the NLS deflection. The maximum uniaxial tension $F_{\max }$ a material can bear without breaking is defined by its cross-sectional area $A_{o}$ and tensile strength $\sigma_{f}$ [22]:

$$
F_{\max }=\sigma_{f} A_{o} .
$$

Given $\left(r_{\text {spring }, x}, r_{\text {spring,y }}\right), \tau_{\max }$, and $\Delta \theta_{\max }$, it is possible to calculate the required uniaxial tension $F_{r e q}$ at a maximum

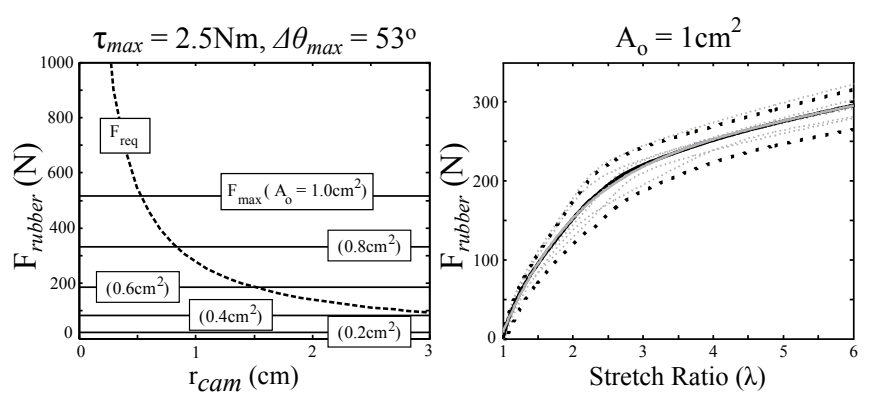

Fig. 3. PMC-770 minimum required tensile force vs. cam radius and tensile test results. L: Minimum required tensile force vs. cam radius to achieve $\tau_{\max }=2.5 \mathrm{Nm}$ for $\Delta \theta_{\max }=53^{\circ}$ using PMC-770. Dashed: Minimum required tensile force. Solid isocontours: maximum tensile force for a given $A_{o}$. R: PMC-770 tensile test results, $A_{o}=1 \mathrm{~cm}^{2}$. Dotted grey: Individual samples $(n=5)$. Solid black: Average of individual samples. Dotted black: Standard deviation of average. Solid grey: Exponential fit of average: $F_{\text {rubber }}(\lambda)=212.1^{0.975 \lambda}-552.5^{-0.938 \lambda} . R^{2}=0.9996$.

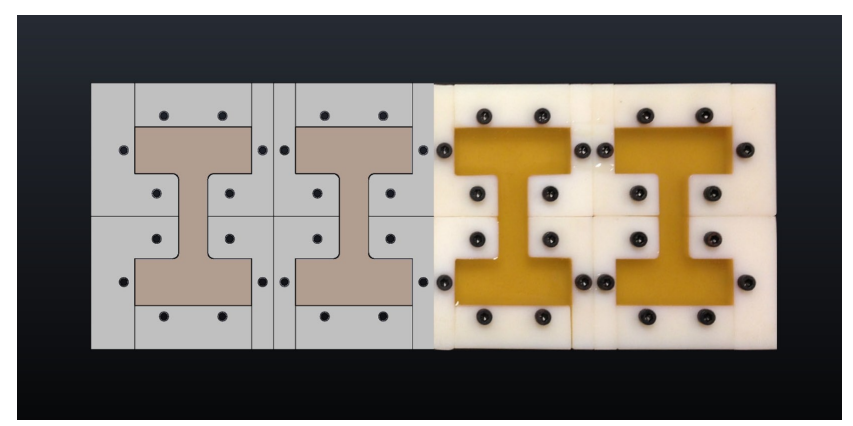

Fig. 4. Puzzle mold. L: Concept R: Rapid prototyped mold with rubber.

cam radius $r_{c a m, \max }$ to realize $\tau_{\max }$ as

$$
F_{\text {req }}=\frac{\tau_{\max }}{r_{\text {cam }, \max } \sin (\beta)}
$$

where $\beta$ is the angle between $\boldsymbol{r}_{\text {cam,max }}$ and $\boldsymbol{F}_{\text {elastic }}$ as shown in figure 1 . With this information, it is possible to select an appropriate $\sigma_{f}$ to realize a desired NLS size. We choose to use a urethane rubber (PMC-770, Smooth-On Inc., $\left.\sigma_{f}=5.17 \mathrm{MPa}\right)$ as our elastic element. Figure 3 plots the relationship between $F_{r e q}, r_{c a m, \max }$, and PMC-770's $F_{\max }$ at various $A_{o}$. We desire an $r_{c a m, \max }$ of $1.7 \mathrm{~cm}$. To account for small imperfections in the manufactured elastic elements, we use a safety factor of $n=3$ when choosing $A_{o}$. Based on this information and our NLS size requirement, we use a rubber elastic element with $A_{o}=1 \mathrm{~cm}^{2}$.

\section{Rubber Manufacturing \& Characterization}

PMC-770 is a two part urethane rubber mixture. The parts are mixed with a planetary centrifugal mixer (AR-100, THINKY USA, Inc.) and cast into rapid prototyped acrylic molds (VeroWhitePlus, Objet Ltd.). Rubber surface defects create stress points during elongation, which can lead to ripping and premature rubber failure. To mitigate damage to cured rubbers during mold extraction, we design multipiece "puzzle molds," which reduce contact area between the rubber and each mold piece compared to a solid-body mold, and make extraction easier (Fig. 4). Rubber samples are cured at room temperature for 24 hours prior to demolding. 


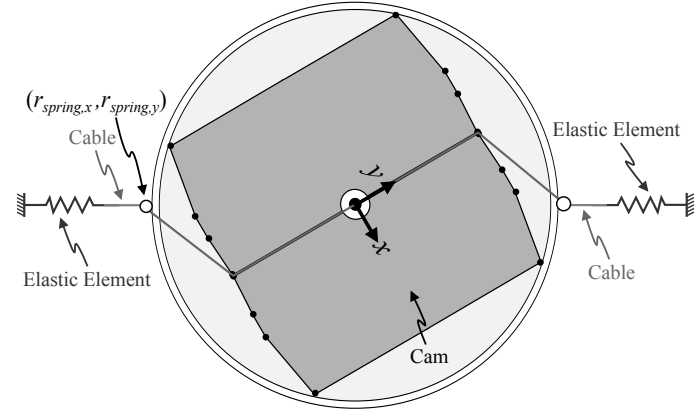

Fig. 5. NLS prototype schematic with optimized cam to realize desired torque profile when using PMC-770 as the elastic element.

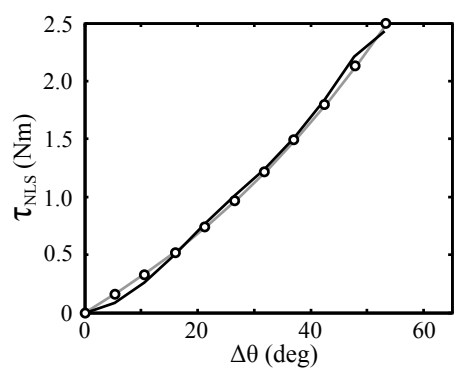

Fig. 6. Desired vs. optimized cam encoded torque-deflection profile. Grey: Exponential torque profile described by equation 6 and parameters in table I. Dots: Discrete torque-deflection pairs used to optimize cam profile. Black: Simulated cam-encoded torque profile.

The force-deflection relationship of the rubber shows both static and velocity-dependent effects. The dominating static effect, the Mullin's Effect, results in rubber softening after a freshly cast sample is stretched to a certain length [17]. This phenomenon occurs as some finite-length polymer chains rupture during initial rubber extension and can no longer resist stretch during subsequent extensions. To account for this effect, samples used to characterize the forcedeflection profile of the rubber are pre-stretched past the maximum elongation that will be observed in the NLS prior to tensile testing. Velocity-dependent effects include strainrate stiffening and hysteresis [16][17]. Both effects are the result of temporary van der Waals bonds forming between polymer chains as they move relative to each other during rubber extension and contraction. Ideally, the rubber's forcedeflection profile should be characterized under nominal operating conditions. Due to velocity limits of the load cell available for tensile testing, we characterize the effect of velocity-dependent factors in subsequent NLS experiments.

Tensile tests are performed with an Instron 4400R Load Frame with a 100lbf analog load cell at an extension rate of 0.5 in $(1.27 \mathrm{~cm})$ per minute. We stretch 5 samples until failure. The relationship between force generated in the rubber $F_{\text {rubber }}$ and nondimensionalized rubber length ("stretch") $\lambda$ is given by

$$
\lambda=\left(L_{o}+\Delta s\right) / L_{o} .
$$

where $L_{o}$ is the unstretched rubber's rest length. An exponential function of the form $F_{\text {rubber }}(\lambda)=a^{b \lambda}+c^{d \lambda}$ is fit to

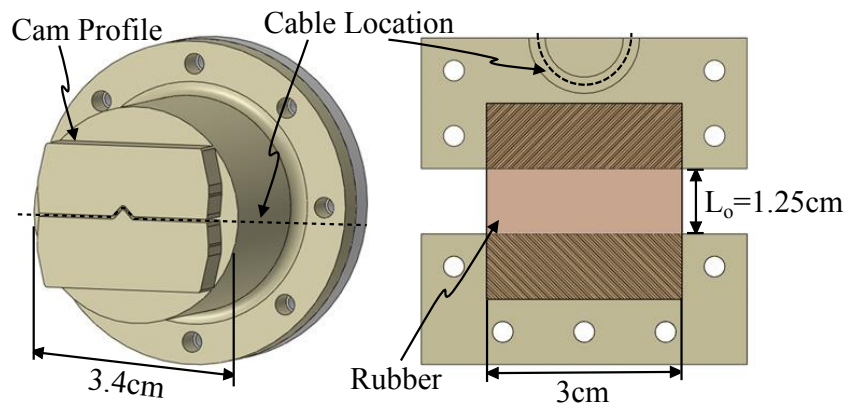

Fig. 7. NLS components. L: CAD rendering of prototyped cam profile. A divot is placed in the middle of the cam to ensure the cable does not slacken. R: Cross-section of retention clamp. Rubber thickness $=3.4 \mathrm{~mm}$ Compression teeth help to secure the rubber during stretching.

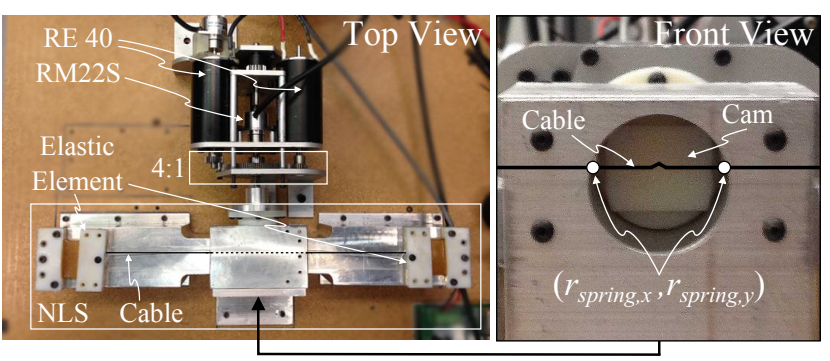

Fig. 8. NLS benchtop setup. L: Top view of benchtop setup. R: Front view close-up of cam.

the average of the tensile data using MATLAB's curve-fitting toolbox (MATLAB cftool) to obtain a nominal force-stretch relationship for the rubber. The results are shown in figure 3. The fit has an $\mathrm{R}$ squared value of $R^{2}=0.9996$.

\section{NLS Prototype}

We find the cam shape of the NLS design shown in figure 2 to realize the desired torque profile in equation 6 using the rubber's empirically characterized force-stretch relationship and the optimization procedure described in section II. For the optimization, we use 11 evenly spaced torque deflection pairs between $\Delta \theta=0$ and $\Delta \theta_{\max }=53^{\circ}$, with an elastic element rest length $L_{o}=1.25 \mathrm{~cm}$. The resulting cam, as well as the simulated torque-deflection profile realized by the cam, are shown in figures 5 and 6 , respectively. The cam encodes the desired torque profile, with a sum squared error of $0.07 \mathrm{Nm}$ and an average error of $0.07 \pm 0.05 \mathrm{Nm}$, equivalent to a $1.4 \%$ of the maximum target torque.

To test the cam profile experimentally, a NLS prototype is printed out of acrylic. Rubber elastic elements are cast into rapid prototyped molds and glued into retention clamps using urethane adhesive (URE-BOND II, Smooth-On Inc.). Polyethylene cable (Solid Spectra, BHP Tackle, $\phi=0.5 \mathrm{~mm}$, $F_{\max }=580 \mathrm{~N}$ ) is used as the cable to interface the cam and rubber elastic element. CAD renderings of NLS components are shown in figure 7.

\section{NLS CHARACTERIZATION}

We test the NLS prototype using the NLS benchtop setup shown in figure 8. Actuator position is controlled using 


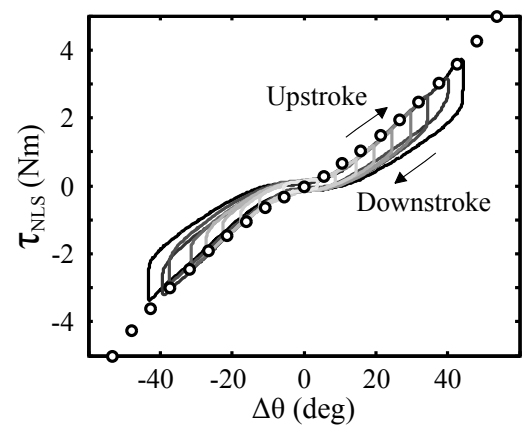

Fig. 9. Measured NLS profile. Dots: Desired torque profile. Solid isocontours: Average torque profile for 15 deflections.
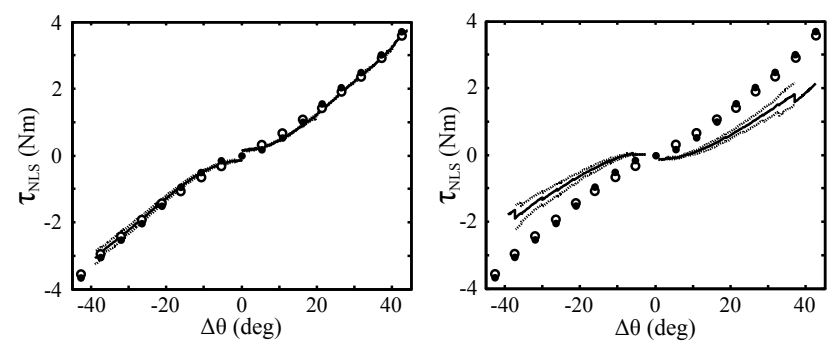

Fig. 10. Measured NLS profile. L: Upstroke. R: Downstroke. Solid: Average for all deflections $(n=150)$. Dotted: Standard deviation of average. White dots: Desired torque profile. Black dots: Cam-encoded torque profile.

Mathwork's xPCTarget software and an EtherCAT motor controller (DZEANTU, Advanced Motion Controls, peak current=20A), which measures applied motor current, from which we calculate spring torque. Spring position is measured with an absolute rotary encoder (RM22S, Renishaw PLC, 13bit), and fed asynchronously to the control PC using a microcontroller (ATmega328-PU, Atmel Corporation). We characterize the spring through three experiments. First, we validate that the cam profile realizes the desired torque profile. Next, we test the NLS' ability to match the desired rise time. Finally, we perform experiments to observe velocitydependent stiffness effects of the rubber elastic element.

\section{A. Torque Profile Validation}

To validate the desired torque profile, the spring is deflected using sinusoidal position commands at a frequency $f=0.1 \mathrm{~Hz}$. The low frequency mitigates potential velocitydependent stiffness effects. 10 sinusoidal position amplitudes are tested with 15 NLS deflections each, where the sinusoid with the largest position amplitude corresponds to the peak current rating of our motor controller. The observed NLS torque-deflection profiles are shown in figure 9. Hysteresis is present between cam engagement of the rubber ("upstroke") and cam disengagement of the rubber ("downstroke"). We quantify NLS performance by analyzing the observed torque profile between NLS upstroke and downstroke separately. Figure 10 plots the average of observed up- and downstroke profiles for all deflections $(n=150)$.

During upstroke, the observed torque profile matches both the desired and cam-encoded torque profile. The average

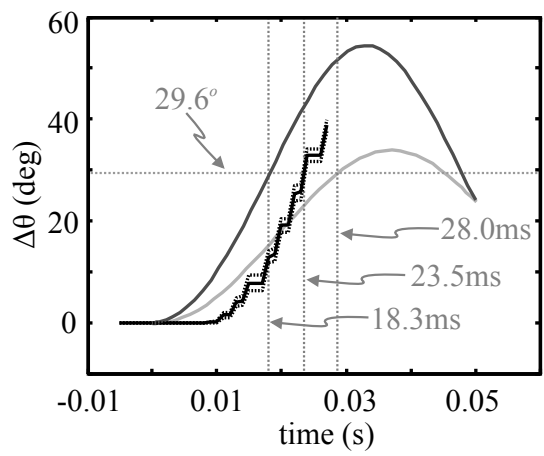

Fig. 11. NLS step response. Solid black: Average step response $(n=10)$. Dotted black: Standard deviation of average. Dark grey: Step response of ideal model. Light grey: Step response of augmented model.

root-mean-squared error between the desired and measured torque profile is $0.10 \mathrm{Nm}$, a relative error of $10 \%$, equivalent to $2 \%$ of maximum target torque. Ideally, we expect no error between the cam-encoded and measured profile. The average root-mean-squared error between the cam-encoded and measured torque profile is $0.11 \mathrm{Nm}$, a relative error of $12 \%$, equivalent to $2.2 \%$ of maximum target torque. Error results from rubber variability between samples. As shown in figure 3 , intersample variation in the force-stretch relationship between rubber samples can be as much as $15 \%$.

During downstroke, hysteretic effects of the rubber result in larger discrepancies between the desired and measured torque profile. We quantify downstroke hysteresis as the average relative error between desired and measured torque. The average relative error between the desired and measured torque is $61 \%$. The average relative error between the camencoded and measured torque is $63 \%$. Compared to other rubbers, the NLS' urethane rubber has large hysteresis [18]. To decrease NLS hysteresis, we plan to investigate use of other rubbers as NLS elastic elements in the future.

\section{B. NLS Step Response}

We next test the NLS' ability to match the desired actuator rise time. For monotonic springs, the correspondence between spring deflection and applied torque is 1:1. The NLS was designed to generate torques up to $5 \mathrm{Nm}$, corresponding to a motor current of $22.7 \mathrm{~A}$, which exceeds the $20 \mathrm{~A}$ peak current rating of our motor controller. Additionally, applying current step changes greater than 10A caused the polyethylene cable to snap. This is a shortcoming of the cable, not the NLS design or rubber. We therefore command a $10 \mathrm{~A}$ step response to the SEA, which corresponds to $\tau_{N L S}=2.2 \mathrm{Nm}$ at $\Delta \theta=29.6^{\circ}$, and compare the time required by the NLS prototype to achieve this deflection with the theoretical rise time predicted by equation 7 . For this torque, the predicted rise time is $18.3 \mathrm{~ms}$, whereas the measured rise time is $23.5 \mathrm{~ms}$, a relative error of $28 \%$ (Fig. 11). The measured response also indicates that slight strain-rate stiffening effects are present in the rubber, seen by the measured response's steeper slope compared to the predicted response. These discrepancies result from the fact that equation 7 used to 


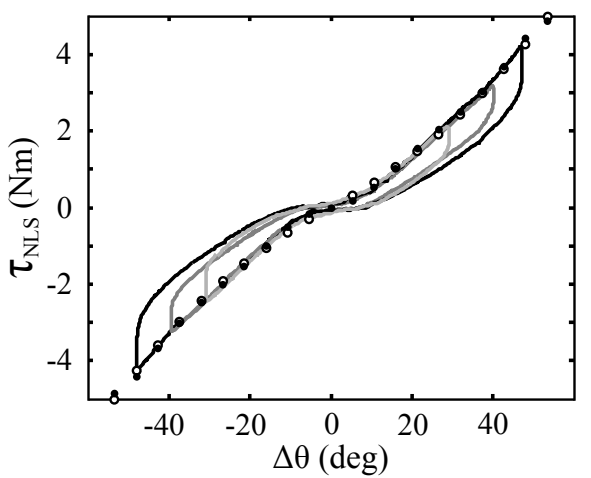

Fig. 12. NLS profile velocity-effects. Black: $f=0.1 \mathrm{~Hz}$. Dark grey: $f=0.5 \mathrm{~Hz}$. Light grey: $f=1.0 \mathrm{~Hz}$.

optimize $\Delta \theta_{\max }$ is an ideal model that does not account for motor and gearing inefficiencies, unmodeled SEA inertia, or rubber strain-rate stiffening effects. Augmenting the model to use better SEA inertia estimates, and motor and gearing efficiencies, $\eta_{\text {motor }}=57 \%$ and $\eta_{\text {gears }}=96 \%$, respectively [23][24], the predicted rise time increases to $28 \mathrm{~ms}$, which is longer than the measured $23.5 \mathrm{~ms}$ rise time (Fig. 11). This faster than predicted rise-time is beneficial for high bandwidth control and again indicates a stiffer than predicted elastic element resulting from velocity-based strain-rate stiffening effects in the rubber.

\section{Velocity-Dependent Stiffness Effects}

To observe velocity-dependent effects, the spring is deflected at three different frequencies $f=0.1 \mathrm{~Hz}, f=0.5 \mathrm{~Hz}$, and $f=1.0 \mathrm{~Hz}$. Higher frequencies could not be tested due to bandwidth limitations of the motor controller's position loop. Resulting torque profiles are shown in figure 12. The experiments indicate no significant velocity-dependent effects on the spring profile when actuating the NLS at these frequencies. Since step response experiments do suggest the presence of stiffening effects when actuating at high frequencies, future experiments, with a higher bandwidth motor controller, will need to be conducted to quantify the relationship between torque profile and actuation speed.

\section{FUTURE WORK}

In this paper, we present an optimization-based synthesis method for compact NLSs and create a NLS prototype that uses nonlinear rubber as the elastic element. With this method, we can design NLSs that realize user-defined nonlinear torque profiles at compact sizes. Two main avenues of future work exist. First, while the results suggest that rubber is a suitable elastic element for NLSs, the type of rubber needs to be carefully chosen to minimize spring hysteresis. To lessen hysteretic effects, we will investigate the use of natural rubbers, though small hysteretic effects may still be present in the resulting NLSs. These effects could be accounted for with a spring state-observer that uses quantified data about the rubber's velocity-dependence and hysteresis to accurately report the transmitted torque, as suggested in [25]. Second, we aim to transfer this technology into our robot's existing SEAs. To make the spring smaller, we will investigate the use of stiffer rubbers.

\section{ACKNOWLEDGMENT}

We thank Carmel Majidi and Lawrence Hayhurst for spring fabrication equipment and William Pingitore for assistance during tensile testing. We also thank Justin Haines and Prasana Velagapudi for help with hardware communication, and Erle Misra and Lauren Finkenauer for design advice throughout the project. Thank you to Christopher Atkeson and Ralph Hollis for their comments on this manuscript.

\section{REFERENCES}

[1] GA Pratt and MM Williamson. Series elastic actuators. Proc IEEE/RSJ IROS, 1:399-406, 1995.

[2] JE Pratt, et al. Series elastic actuators for high fidelity force control. Industrial Robot, 29(3):234-241, 2002.

[3] NG Tsagarakis, et al. A compact soft actuator unit for small scale human friendly robots. Proc IEEE ICRA, 1:4356-4362, 2009.

[4] KC Kong, et al. A compact rotary series elastic actuator for human assistive devices. IEEE/ASME Trans Mechatron, 17(2):288-297, 2012.

[5] JF Veneman, et al. A series-elastic and Bowden-cable-based actuation system for use as a torque actuator in exoskeleton-type robots. IJRR, 25(3):261-281, 2006.

[6] S Wolf and G Hirzinger. A new variable stiffness design: Matching requirements of the next robot generation. Proc IEEE ICRA, 1:17411746, 2008.

[7] DW Robinson. Design and analysis of series elasticity in closed-loop actuator force control. PhD Thesis, MIT, 2000.

[8] JW Hurst, et al. An actuator with physically variable stiffness for highly dynamic legged locomotion. Proc IEEE ICRA, 5:4662-4667, 2004.

[9] G Tonietti, et al. Design and control of a variable stiffness actuator for safe and fast physical human/robot interaction. Proc IEEE ICRA, 1:526-531, 2005.

[10] R van Ham, et al. MACCEPA, the mechanically adjustable compliance and controllable equilibrium position actuator: Design and implementation in a biped robot. Rob and Aut Sys, 55:761-768, 2007.

[11] A Jafari, et al. A novel actuator with adjustable stiffness (AwAS). Proc IEEE/RSJ IROS, 1:4201-4206, 2010.

[12] SA Migliore, et al. Biologically inspired joint stiffness control. Proc IEEE ICRA, 1:4508-4513, 2005.

[13] SA Migliore, et al. Novel nonlinear elastic actuators for passively controlling robotic joint compliance. J Mech Des, 129(4):406-412, 2007.

[14] N Schmit and M Okada. Design and realization of a non-circular cable spool to synthesize a nonlinear rotational spring. Adv Rob, 26:235-252, 2012.

[15] N Schmit and M Okada. Optimal design of nonlinear springs in robot mechanism: simultaneous design of trajectory and spring force profiles. Adv Rob, 27:33-46, 2013.

[16] AN Gent, et al. Engineering with rubber: How to design rubber components, 2nd ed. Hanser Publications, 2001.

[17] JT Bauman. Fatigue, stress, and strain of rubber components: A guide for design engineers. Hanser Publications, 2008.

[18] D Rollinson, et al. Design and modeling of a series elastic element for snake robots. Proc ASME DSCC, 2013.

[19] F Karlsson and A Persson. Modelling non-linear dynamics of rubber bushings - parameter identification and validation. MS Thesis, Lund University, 2003.

[20] N Hansen. CMAE-ES MATLAB implementation (Version 3.61) [Computer program]. Available at https://www.lri.fr/ hansen/cmaes.m

[21] A Schepelmann, et al. Development of a testbed for robotic neuromuscular controllers. Proc RSS, 2012.

[22] JM Gere. Mechanics of Materials, 6th ed. Brooks/Cole, 2004.

[23] Maxon Motor. RE40 Datasheet. Maxon Program 2010/2011, 2010.

[24] CE Wilson and JP Sadler. Kinematics and Dynamics of Machinery, 3rd ed. Prentice Hall, 2003.

[25] F Parietti, et al. Series viscoelastic actuators can match human force perception. IEEE/ASME Trans Mechatron, 16(5): 853-860, 2011. 